\title{
A Hybrid Common Mode Choke Optimization Method for Input Line EMI Filter Design
}

\author{
Sofiane Khelladi ${ }^{1}$, Khadidja Saci ${ }^{1}$, Abdelchafik Hadjadj $^{1}$, and Achour Ales ${ }^{2}$ \\ ${ }^{1}$ LACOSERE laboratory, Electrical Engineering Department, Amar Telidji University of Laghouat, Algeria \\ ${ }^{2}$ Department of Electromagnetic Compatibility, Polytechnic Military School: Bordj El Bahri, Algiers,Algeria
}

\begin{abstract}
In this paper, an optimization method of toroidal core-based Hybrid common mode chokes (HCMCs) for the design of an Electromagnetic interferences (EMI) filter is proposed. A dedicated algorithm is developed using MATLAB to characterize compact HCMCs that exhibit effective CommonMode $(\mathrm{CM})$ chokes with optimized leakage inductances by systematic variations in the winding patterns and geometric dimensions of their magnetic cores. It takes into consideration the physical limitations of these components as well as the constraints related to the design of EMI filters. The proposed algorithm allows through a small computational task to propose a variety of configurations for optimal HCMCs. Finite element method (FEM) simulations are conducted on the HCMCs to extract their CM and leakage inductances. The results are compared with those calculated analytically and yield a good match. The performances of optimized HCMCs are evaluated through their implementation in the designed filter. All the cases of HCMCs including the smallest one allow the EMI filter to easily qualify a power converter to an electromagnetic compatibility (EMC) standard.
\end{abstract}

INDEX TERMS Electromagnetic interferences filter (EMI), Hybrid common mode choke (HCMC), Optimization algorithm.

\section{INTRODUCTION}

$\mathbf{T}$ HE proliferation of switch mode power supplies (SMPS) and their wide usage in all modern industrial and domestic energy applications are attributed to their high performances, compactness, and competitive prices . However, Electromagnetic Compatibility (EMC) regulations require on these power devices to meet specific standards due to their high induced levels of electromagnetic interferences (EMI) mainly conducted Common-Mode (CM) and Differential-Mode (DM) noises. This usually necessitates the implementation of passive EMI filters as they present the main countermeasure in the EMC arsenal against EMI due to their effective mitigation performance and their basic and low-cost conception process. On the other hand, EMI filters occupy a substantial portion of power converter volume and weight (up to 30\%) [1]. This comes back to the size of their CM and DM chokes in particular CM chokes (CMCs) which are usually the bulkiest filter components. Therefore, to achieve the design of compact and effective EMI filters many optimization methods and integration techniques were vastly explored to reduce the size/number and weight of these components. In [2], the leakage inductance of a toroidal CMC is improved by inserting a smaller DM toroidal coil within. Although this structure reduces the volume of the filter, it significantly heightens the total parasitic capacitance of the resulting choke. Similar to this approach, in [3] the leakage inductance of a toroidal CMC is increased by inserting the latter into an EQ core made of MnZn ferrite, which enhances the DM filtering but still, the resulting structure will exhibit additional size and weight. Another approach described in [4] suggests coating the outside of the CMC with a magnetic epoxy mixture which enhanced the leakage inductance. Yet, such component requires higher labor cost because of the fabrication and the coating process of the magnetic epoxy mixture. Moreover, in [5] integration of a CM and DM inductor into a single magnetic core unit using EE-type and EIE-type cores was presented. This method helped a $69 \%$ reduction of the inductor size for single-stage EMI filter but still, this type of cores has a limited capacity of heat dissipation which affects their efficiency compared to discrete inductors. The work described in [6] proposed the computation of the optimal volume of a toroidal $\mathrm{CM}$ inductor taking into account the problems related to the saturation of its core and the overlapping of the coil turns. This method achieved an optimal size for the $\mathrm{CM}$ inductor by providing a useful computation tool. The most frequently employed CMCs in commercial EMI filters are still constructed on toroidal cores owing to their low cost and wide availability according to [7]. A toroidal CMC is usually realized with coupled inductors 
whereas the DM inductance is achieved depending on the amount of required DM filtering either by actual discrete inductors or by the CMC parasitic leakage inductance. This inductance depends on the CMC structure and has no physical size which limits the number of components and mounting area. Furthermore, since this inductance is an air-cored inductor, it never saturates at any level of current keeping sustainable filtering performance which favors the usage of a toroidal CMC for CM and DM noise suppression. This inherent parasitical parameter has been addressed in several research papers, either as a constraint in the design of CMCs which is the case in [8]-[10] or to act as a formal DM inductor in EMI filters design as in [11]-[18]. References [19], [20] reported the designable parameter for a CMC. This parameter defines the CM and DM impedances of the CMC as well as its parasitic capacitances. The CMC DM impedance which improves the DM noise filtering is defined by the CMC dimensions, the number of turns and the angle of its leakage inductances. However, due to their complex mechanical structures, toroidal CMCs provide leakage inductances with limited values ranging from 0.1 $\%$ to $2 \%$ of their total values depending on the winding system. The CMCs with sectional winding exhibit a higher leakage inductance compared to CMCs with bifilar winding [21]. Taking into account the features that this stray element offers, the concept of exploiting the leakage inductance of a standard toroidal CMC remains tempting compared to the aforementioned methods.

This paper presents an effective optimization approach intended for the design of EMI filters with compact and effective CMCs with optimized leakage inductances. The paper is structured as follows. Section II describes the design steps of the EMI filter. The HCMC optimization method is detailed in section III. Section IV is devoted to the application of the proposed optimization method in the design of an EMI filter dedicated to a power conversation system. Section V presents discussion. The conclusion of this work is given in section VI.

\section{THE EMI FILTER DESIGN PROCEDURE}

\section{A. SIZING OF THE EMI FILTER}

The EMI filter is constructed to fulfill the EMC standard requirements imposed on power converters by sizing its elements to provide the required mitigation of EMI. The design of the filter is based mainly on the levels of EMI disturbances and the EMC Standard that they are intended to qualify for. The proper sizing of the filter components is governed by several constraints related to power density, safety regulations, and the stability of the power electronic system. For the CM filter parameters, the value of its capacitors is limited by safety standards which define the maximum leakage current to earth thus, imposing the need to compensate by increasing the $\mathrm{CM}$ inductors value and thereby their sizes. The sizing of the DM filter parameters depends on the amount of DM noise to be attenuated taking into account the impact of its components impedance on the stability of the power conversion system. The design workflow of the EMI filter is summarized in the diagram shown in Fig. 1 including the optimization algorithm as an essential step to provide the recommendations for optimal HCMCs. The filter design procedure requires predefined parameters according to EMI requirements i.e.:

- The EMC standards that EMI are intended to meet (e.g. EN 55032 analog to CISPR 32);

- The conducted CM and DM EMI levels induced by the power conversion system $\left(\mathrm{V}_{\mathrm{CM}}\right.$ and $\left.\mathrm{V}_{\mathrm{DM}}\right)$;

- The required attenuations of the CM and DM conducted EMI (A $A_{\text {CM_req }}$ and $A_{D M}$ req $)$;

- The EMI filter configuration (e.g., $\Gamma, \mathrm{T}$ or $\Pi$ );

- The CM and DM filters cutoff frequencies $\left(f_{o_{-} \mathrm{CM}}\right.$, $f_{o_{-} \mathrm{DM}}$ ).

The conducted CM and DM noises are evaluated separately to establish the key points in their frequencies spectra with respect to EMC limits and define the required $\mathrm{CM}$ and DM attenuations indicated in (1) and (2) as :

$$
\begin{aligned}
& A_{C M_{-} r e q}=V_{C M}[d B \mu V]-V_{\text {Limit }}+S M \\
& A_{D M_{-} r e q}=V_{D M}[d B \mu V]-V_{\text {Limit }}+S M
\end{aligned}
$$

Where $\left(\mathrm{V}_{\text {Limit }}\right)$ is the limit defined by the standard and $\mathrm{SM}$ is a safety margin usually set to $6 \mathrm{~dB}$ to account for possible inaccuracies in conducted EMI estimation and stray inductive and capacitive components [22].

The filter topology is considered as a preliminary step in the filter design (e.g., $40 \mathrm{~dB} / \mathrm{dec}$ for $\Gamma$ type L-C singlestage, $60 \mathrm{~dB} / \mathrm{dec}$ for $\Pi$ or $\mathrm{T}$ type $\mathrm{L}-\mathrm{C}$ single-stage... etc.). Accordingly, $f_{O_{-} \mathrm{CM}}$ and $f_{o_{-} \mathrm{DM}}$ are delivered by (3):

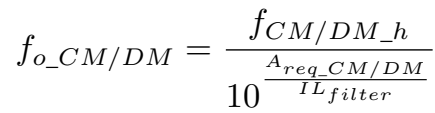

where $f_{o_{-} \mathrm{CM}} / \mathrm{DM}_{-} \mathrm{h}$ are the frequencies of the CM/DM harmonic components to be attenuated. $\mathrm{IL}_{\mathrm{filter}}$ is the theoretical insertion loss of the filter depending on its topology [15]. With $f_{o_{-} \mathrm{CM} / \mathrm{DM}}$ being defined, the required components forming the CM and DM sections of the filter are determined as in (4) and (5):

$$
\begin{array}{ll}
L_{C M_{-} r e q}=\frac{1}{C_{C M}\left(2 \pi f_{o_{-} C M}\right)^{2}} & C_{C M}=2 C_{Y} \\
L_{D M_{-} r e q}=\frac{1}{C_{D M}\left(2 \pi f_{o_{-} D M}\right)^{2}} & C_{D M}=C_{X 1}=C_{X 2}
\end{array}
$$

$\mathrm{L}_{\mathrm{CM}}$ req and $\mathrm{L}_{\mathrm{DM}}$ req are respectively the filter $\mathrm{CM}$ and $\mathrm{DM}$ required inductances, whereas $\mathrm{C}_{\mathrm{CM}}$ and $\mathrm{C}_{\mathrm{DM}}$ are the respective capacitances of its $\mathrm{CM}$ and $\mathrm{DM}$ sections. The design worflow of the filter is done with the following steps:

- The pairs of components $\left(\mathrm{L}_{\mathrm{CM}_{-} \text {req }}, \mathrm{C}_{\mathrm{Y}}\right)$ and $\left(\mathrm{L}_{\mathrm{DM}}\right.$ req , $\mathrm{C}_{\mathrm{X}}$ ) that achieve the minimum required insertion losses profiles are determined according to EMI requirements where the filter $\mathrm{CM}$ and $\mathrm{DM}$ caps $\left(\mathrm{C}_{\mathrm{Y}}, \mathrm{C}_{\mathrm{X}}\right)$ selection is done beforehand then their respective inductors ( $\mathrm{L}_{\mathrm{CM} \_ \text {req }}, \mathrm{L}_{\mathrm{DM}}$ _req $)$ are calculated accordingly; 
- Based on the calculated pair of inductors $\left(\mathrm{L}_{\mathrm{CM} \_ \text {req }}\right.$, $\left.\mathrm{L}_{\mathrm{DM} \_ \text {req }}\right)$ the proposed algorithm will define the best possible configurations of HCMCs with their feasible pairs of effective CM inductances and adequate leakage inductances taking into account their physical and geometric limitations;

- The optimal HCMCs are chosen among the other cases in terms of minimum volume;

- The geometric parameters of optimized HCMCs are used as recommendations to develop 3D prototypes then FEM simulations are carried out on each of them separately after including the material database of their magnetic cores;

- The performances of optimized HCMCs are evaluated through their implementation in the designed filter model to qualify a power converter to an EMC standard.

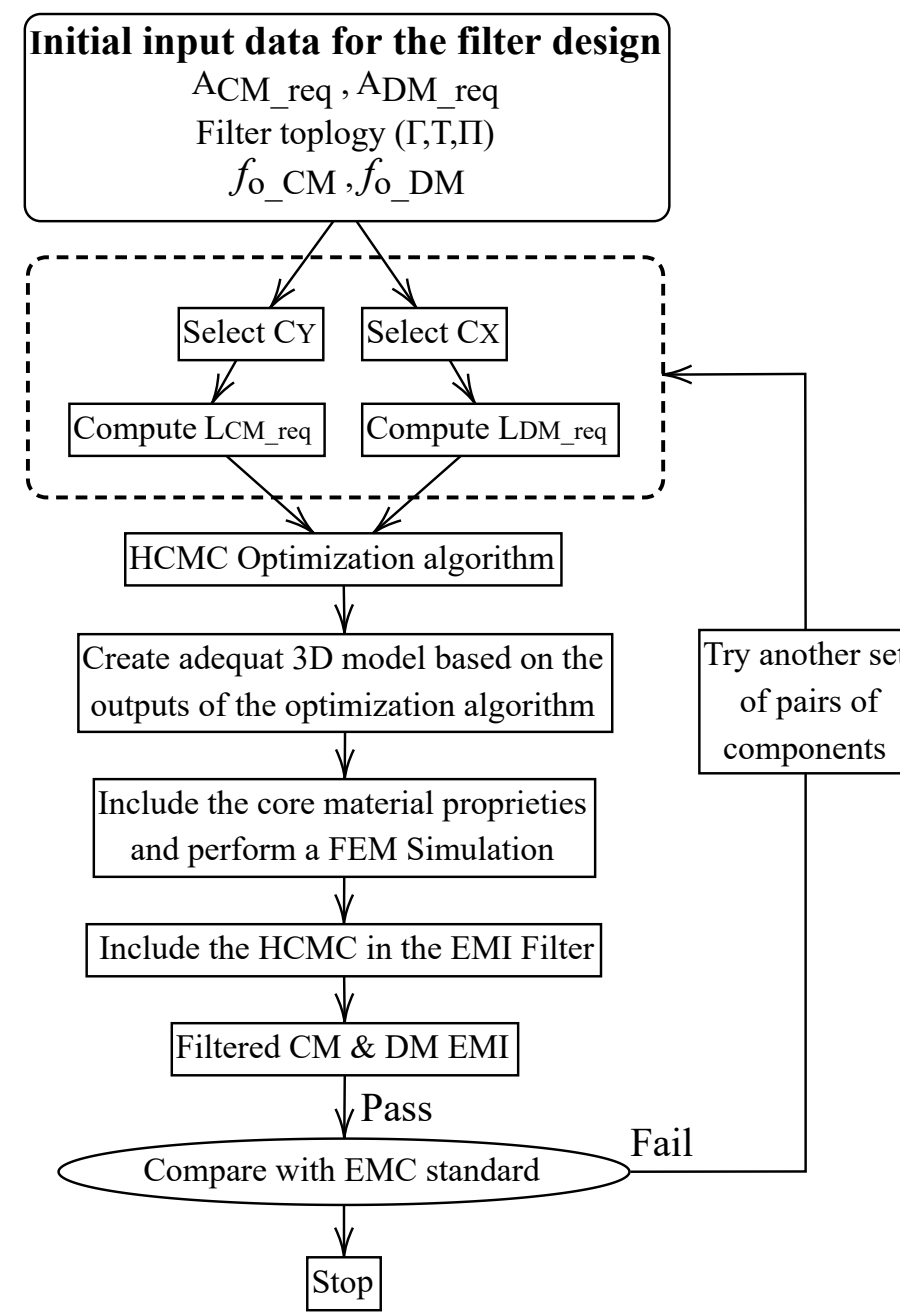

FIGURE 1: Diagram of the EMI filter design

\section{B. 3-D FEM SIMULATION OF THE HCMC}

Three-dimensional (3-D) FEM simulations offer a powerful means to analyze electrostatic and electromagnetic problems linked to different power components and allow to model their behavior at high frequencies which is suitable for the characterization of CMCs. Usually, a proper adaptation of large or complex problems for a 3-D FEM simulation requires a certain degree of simplification to be made on the model's structure to gain in simulation time and memory at the expense of accuracy. Considering this, the optimized HCMCs which reflect as close as possible the geometry of real choke (Fig. 2d) are modeled and treated separately. The choice of the magnetic material is done beforehand by defining its main characteristics namely its real and complex magnetic permeability $\left(\mu^{\prime}, \mu^{\prime \prime}\right)$ in the material model relative to the toroidal core. The 3-D FEM simulations performed on the HCMCs models took an average computational time of roughly $45 \mathrm{~min}$ for each case using a server comprising a CPU with 6 cores running each at $2.20 \mathrm{GHz}$ and a $16 \mathrm{~GB}$ DDR4 RAM.

\section{PROPOSED OPTIMIZATION METHOD OF THE HCMC A. THE HYBRID COMMON MODE CHOKE}

The CMC is the centerpiece in EMI filters and take a crucial part in their design. Its formed of two windings wound around a highly permeable core in the same direction for single-phase applications as shown in Fig. 2a. The magnetic field $\mathrm{H}_{\mathrm{CM}}$ induced by $\mathrm{CM}$ currents is cumulated within the toroidal core creating an impedance that acts against these currents. However, in a CMC, the leakage fluxes caused by the DM currents do not fully cancel out which creates magnetic leakage fields $\mathrm{H}_{\text {leak }}$ on each coil of the CMC (bleu curves) and defines its parasitic leakage inductance [23]. Constructively, in the design of filters, this inherent parasitical parameter has been exploited by designers to act as a regular DM inductor which introduced the concept of HCMC. In Fig. $2 b, d$ is the wire diameter estimated with respect to the power system rated current, $e$ is the wire insulation thickness, and $\theta$ is the angular coverage of each winding. The CMC geometric parameters are shown in Fig. 2c where ID and OD are the inner and outer diameters of the core respectively whereas $\mathrm{H}$ is its height. Fig. $2 \mathrm{~d}$ shows the 3D model of a CMC.

\section{B. THE HCMC CM INDUCTANCE}

The sizing of a single-phase HCMC inductance to handle the CM and DM currents increase the design considerations due to the physical limits related to this device including saturation, high frequency operation, and others related to its geometric aspect. These considerations can be addressed by setting constraints on the design of this component to obtain its best performances. A single-phase CMC inductance is delivered by (6) as:

$$
L_{C M}(f)=N^{2} \mu(f) A_{e} / l_{e}
$$

where $\mu$ is the permeability of the core material. $A_{e}$ and $l_{e}$ are the effective cross-sectional area and the mean path length of the magnetic core respectively. 

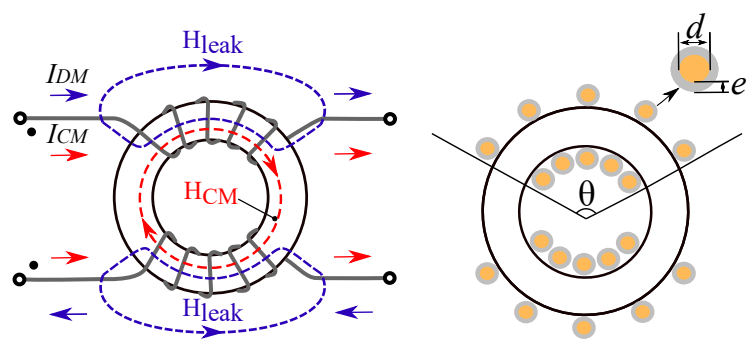

(a)

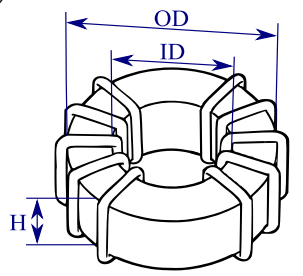

(c)

(b)

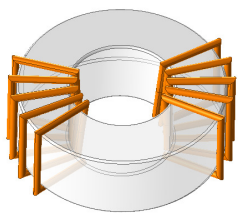

(d)

FIGURE 2: (a) Sectional wound CMC, (b) Cross-sectional view of the CMC showing the toroidal core window area, (c) Dimensions of the CMC, (d) 3D model of the CMC

CMCs are built on magnetic cores destined to exhibit a good dissipative behavior against the noise signals energy and the overall size of these magnetic cores is related to their capacity to handle this energy. Moreover, the core's permeability is strongly dependent on frequency, and knowing that its typical curves decrease with frequency for ferrite and nanocrystalline core materials, the design of a specific inductance that maintains its value at high frequency imposes the increase of the core size and/or the number of turns $\mathrm{N}$ according to (6). The nonsaturation limit of the core is set by ensuring that the maximum magnetic field in the inductor $H_{\max }$ is always beneath the saturation magnetic field $H_{\text {sat }}$ of the used material as illustrated in (7):

$$
H_{\max }=\frac{N I_{\max }}{2 \pi r} \leq H_{\text {sat }}
$$

where $\mathrm{I}_{\max }$ is the maximum CM current and " $r$ " is the inner radii of the toroidal core.

For a single layer CMC, a high number of turns $\mathrm{N}$ will cause the saturation of the core according to (7) and at the same time raise the problem of the winding that must fit through the core window. Hence to avoid saturation and guarantee the effective usage of the core window area there are two design constraints to ensure when sizing the $\mathrm{CM}$ inductor. The constraint on the saturation is verified with respect to ID taking into consideration (7) and it's defined as in (8):

$$
I D \geq \frac{I_{\max }}{\pi H_{\text {sat }}} N
$$

Secondly, when designing a CM inductor on a toroidal core the effective use of window area implies enough space left between windings of different phases. It also imposes to ensure that the required number of turns will fit on onehalf of the core as it has to be enough angular spacing between the turns of the windings to decrease the effect of the parasitic capacitances [24]. Thus, the number of turns included in (8) needs also to be included in the following relationship which defines the minimum inner diameter of the core that fulfills the condition for effective use of window area.

$$
I D \geq \frac{2(d+2 e)}{\theta} N+\frac{d+e}{2}
$$

To acquire the proper $\mathrm{CM}$ filtering the theoretical value of $\mathrm{L}_{\mathrm{CM}}$ for the designed HCMC should attain an effective value always equal or above the required inductance defined according to EMI specifications. For an input line filter with a given $\mathrm{CM}$ attenuation, a higher value of $\mathrm{L}_{\mathrm{CM}}$ in a given $\left(\mathrm{L}_{\mathrm{CM}}, \mathrm{C}_{\mathrm{Y}}\right)$ pair will push the filter cutoff frequency towards low frequencies which improves furthermore the attenuation of the filter and in the same time diminish the $\mathrm{CM}$ currents at low frequencies. Hence, the designed HCMC CM inductance must achieve (10).

$$
L_{C M} \geq L_{C M \_r e q}
$$

with $\mathrm{L}_{\mathrm{CM}}$ determined by (6) and $\mathrm{L}_{\mathrm{CM} \_ \text {req }}$ as the minimum required $\mathrm{CM}$ inductance defined from the $\mathrm{CM}$ filter design specifications.

\section{THE HCMC LEAKAGE INDUCTANCE}

The unwounded section of the core evaluated in degrees is one of the parameters affecting directly the leakage inductance of a toroidal core-based CM choke according to [25]. Based on the methodology described in [26] the calculation of the leakage inductance of a single-phase CM inductor is done by multiplying the effective mean path length of the leakage path of each phase $l_{e f f}$ by the inductance of an airgap toroid. Presuming that the leakage magnetic field paths follow the blue lines shown in Fig. 2a, $l_{e f f}$ is empirically derived for windings which span a section of the core above $60^{\circ}(\theta>\pi / 3)$ as in (11):

$$
l_{\text {eff }}=l_{e} \sqrt{\frac{\theta}{2 \pi}+\frac{1}{\pi} \sin \frac{\theta}{2}}
$$

The leakage inductance of each phase can be therefore estimated with (12):

$$
L_{\text {leak }}=2.5 \mu_{0} N^{2} \frac{A_{e}}{l_{\text {eff }}}\left(\frac{l_{e}}{2} \sqrt{\frac{\pi}{A_{e}}}\right)^{1.45}
$$

Considering a specific core size and taking into account (12), an increased number of turns is one way to exploit a larger leakage inductance, but this will require a small wire size that limits RMS currents. Such a manner will also cause high winding losses, temperature rise and will expose the magnetic core to saturation as mentioned before . Conversely, a low number of turns $\mathrm{N}$ will lead to small leakage inductance. Another approach will be to enlarge the core, but this will result in large and high-cost filters. Hence, the control of saturation and the proper amount of leakage inductance impose a compromise in the design of 
the HCMC. Furthermore, the stability of power electronic systems invokes the Middlebrook criterion for input filters illustrated through (13) as:

$$
Z_{o}=\sqrt{\frac{L_{D M_{-} \max }}{C_{X}}} \ll Z_{\text {in }}
$$

This latter impose on the output impedance of the input DM filter $Z_{o}$ to be much lower than the power converter input impedance $Z_{\text {in }}$ thus, bounding the allowed values of the required $D M$ inductor $L_{D M}$ req to a maximum value denoted as $\mathrm{L}_{\mathrm{DM} \_ \text {max }}$.

The feasible pairs of the DM filter components $\left(\mathrm{L}_{\mathrm{DM}}, \mathrm{C}_{\mathrm{X}}\right)$ which verify (13) are defined in the allowed ranges of values determined with the intersection of $Z_{o}$ and $f_{o_{-} \mathrm{DM}}$ axes in a reactance graph. Given the substitution of the DM inductor with the CMC leakage inductance which doesn't exhibit any physical size, the choice of a minimum value of $\mathrm{C}_{\mathrm{X}}$ is preferred to gain the highest value of leakage inductance. Accordingly, the HCMC will be designed to harness the highest value of leakage inductance on each winding defined as in (14).

$$
L_{\text {leak }} \leq \frac{1}{2} L_{D M_{-} \max }
$$

\section{THE HCMC VOLUME}

Improving power density imposes a high-frequency operation with compact size in the design of any component and knowing that inductors are typically the largest components in electronic power systems, the key to small size will be the use of smaller inductors. Accordingly, the global volume $\mathrm{V}$ of the HCMC mainly contributed by the magnetic core size in addition to wire size is evaluated with (15) as follow:

$$
V=\pi(H+2(d+e))\left(\frac{O D}{2}+d+e\right)^{2}
$$

\section{E. DEVELOPMENT OF THE OPTIMIZATION PROSSES}

The sizing of the HCMC for a given CM and DM filters impose on its pair of inductances $\left(\mathrm{L}_{\mathrm{CM}}, \mathrm{L}_{\text {leak }}\right)$ the constraints predefined with (10) and (14) respectively. The constraints on the saturation and the proper use of the window area of the core are verified with respect to ID through (8) and (9) respectively. The geometrical aspect of the HCMC which is affected by all these constraints gets reflected on the total volume of the HCMC evaluated with (15). The optimization algorithm of the HCMC taking into account all the constraints mentioned before is described through the flow chart illustrated in Fig. 3. The development of this chart is done with the following steps:

- First, the input data are to be determined:

- The maximum operating current $\mathrm{I}_{\text {phase_max; }}$

- The maximum CM current $I_{\max }$;

- The minimum required $\mathrm{CM}$ inductance $\mathrm{L}_{\mathrm{CM}}$ req and the maximum allowed DM inductance $\mathrm{L}_{\mathrm{DM} \_ \text {max }}$;

- The chosen magnetic material database $\mu(f)$, $\mathrm{H}_{\mathrm{sat}}$;
- Select wire diameter with respect to $\mathrm{I}_{\text {phase_max; }}$;

- Perform an iterative variation of ID, OD, $\mathrm{H}$, and $\theta$ alongside $\mathrm{N}$ which is varied to exploit the increase in the number of turns for a smaller magnetic core;

- Check every combination of the varied parameters against the constraints defined with (8) then (9) respectively;

- Evaluate $\mathrm{L}_{\mathrm{CM}}$ and $\mathrm{L}_{\text {leak }}$ respectively for every case approved by the previous step then check against the conditions set with (10) then (14) respectively;

- Evaluate with (15) the volume V for every case approved by the previous step;

- The output parameters ID, OD, H, $\theta, \mathrm{N}$, for all approved cases are considered as recommendations for the best HCMCs in terms of their inductances $\left(\mathrm{L}_{\mathrm{CM}}\right.$, $\mathrm{L}_{\text {leak }}$ ) and their volumes.

These geometric parameters are used to develop 3D prototypes of the HCMCs then FEM simulations are carried out on each of them separately. The simulation results of their respective inductances $\mathrm{L}_{\mathrm{CM}}$ and $\mathrm{L}_{\text {leak }}$ are then compared with those computed analytically.

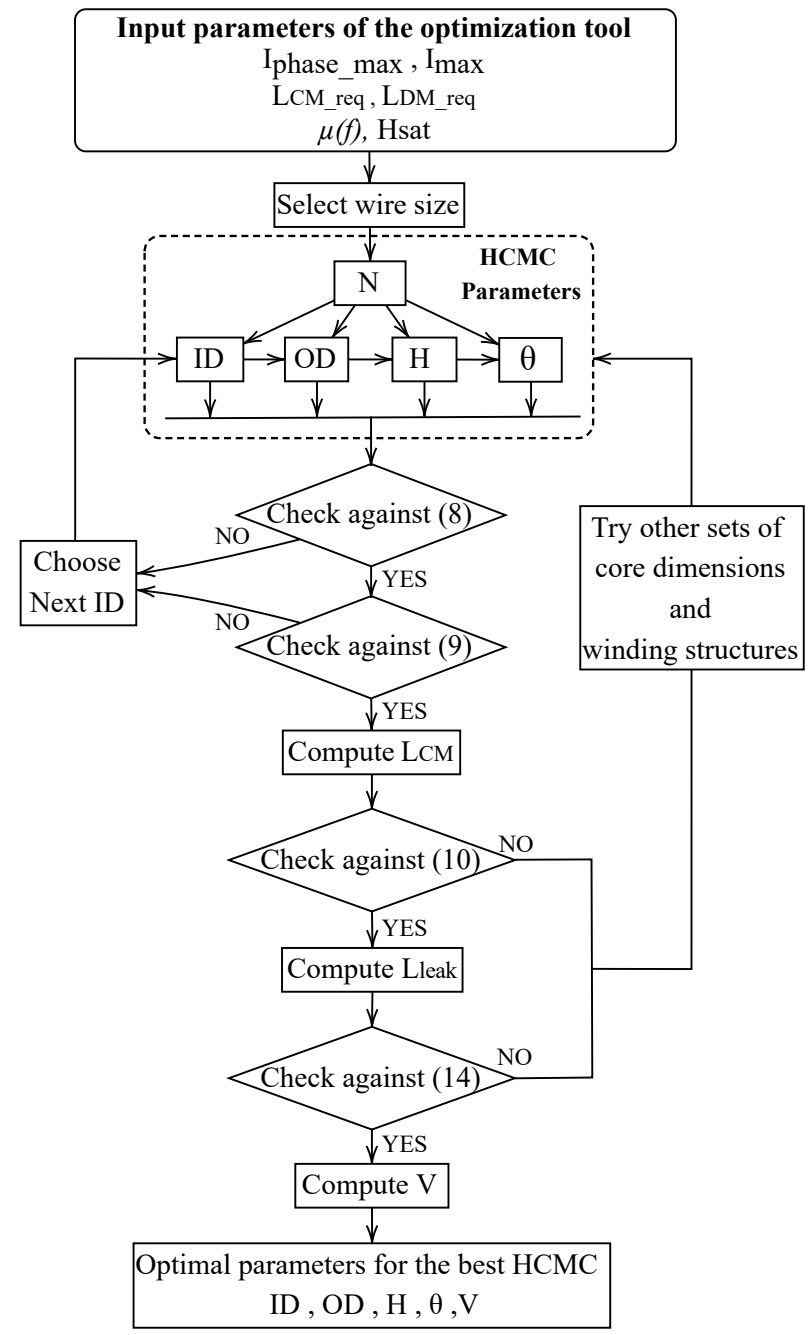

FIGURE 3: Flow chart of the HCMC optimization algorithm 


\section{APPLICATION OF THE PROPOSED METHOD}

The strength and the feasibility of the proposed design method are demonstrated through an EMI filter designed for a DC-DC conversion system described in [27]. It comprises a standard and basic switching cell (Buck converter) working in continuous conduction mode (CCM) at a switching frequency $f_{s w}=20 \mathrm{kHz}$ with a duty cycle $D=50 \%$. The converter is connected to the supply main $\mathrm{V}_{\mathrm{DC}}$ through a line impedance stabilization network (LISN). The conducted EMI levels related to the power conversion system shown in Fig. 4a are evaluated against the EMC standard EN 55032. The results presented in Fig. 5 allow us to determine the key points in the CM and DM spectra and define the required input data for the design of the filter. The adopted filter arrangement is illustrated in Fig. 4b. The CM section of the filter includes a coupled inductor $\mathrm{L}_{\mathrm{CM}}$ and $\mathrm{Y}$ capacitors $\left(C_{Y 1}\right.$ and $\left.C_{Y 2}\right)$ used to shunt each line to the ground. The DM section includes two DM capacitors $\left(C_{X 1}\right.$ and $\left.C_{X 2}\right)$ alongside the leakage inductance of the HCMC which acts as two DM inductors in the line and neutral to form the DM filter. The filter $\mathrm{CM}$ capacitors are set to $\left(C_{Y 1}=\right.$ $C_{Y 2}=10 \mathrm{nF}$ ) which gives $L_{C M_{-} r e q}=108 \mu \mathrm{H}$ whereas the DM filter capacitors were set to $\left(C_{X 1}=C_{X 2}=2.2 \mu F\right)$ which yields $L_{D M_{-} \max }=5 \mu \mathrm{H}$ and allowed to harness the highest value of $L_{\text {leak }}(2.5 \mu \mathrm{H}$ per phase) for the designed HCMC. For this application, the wire diameter is defined to be $d=0.7 \mathrm{~mm}$. The N30 material manufactured by EPCOS with initial permeability of 4300 and saturation flux of $0.38 \mathrm{~T}$ is used for the magnetic cores.

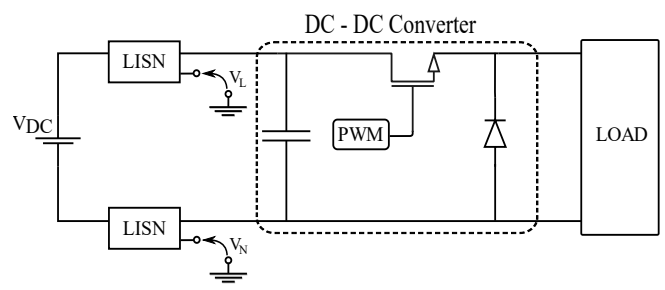

(a)

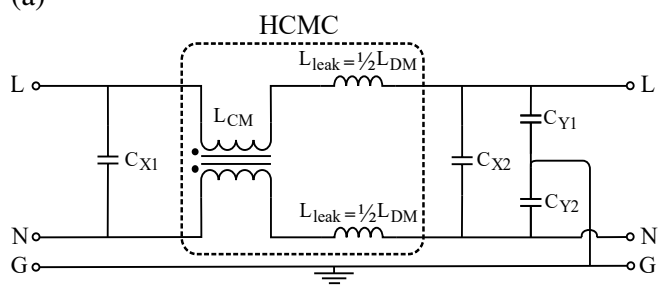

(b)

FIGURE 4: The considered power conversion system and the EMI filter designed for it. (a) Simulation model of the DC-DC system conducted emissions, (b) Typical singlestage filter configuration

\section{DISCUSSION}

\section{A. EVALUATION OF THE OPTIMIZATION METHOD}

Table I presents 13 cases of HCMCs evaluated against the predefined constraints with a fixed number of turns $\mathrm{N}$ and

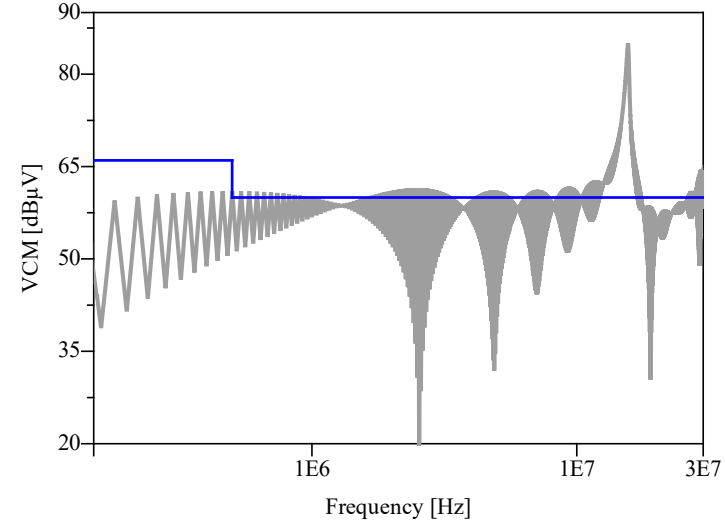

(a)

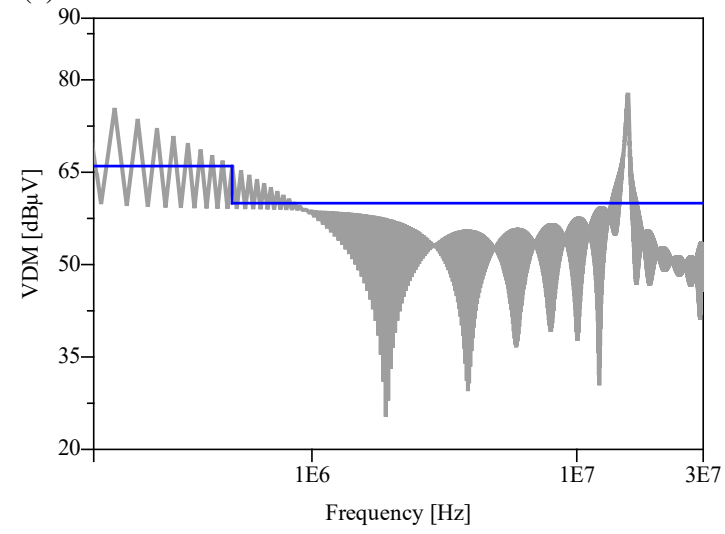

(b)

FIGURE 5: The Conducted EMI levels induced by the power converter without an input filter (a) CM noise (b) DM noise

varying ID, $\mathrm{OD}, \mathrm{H}$, and $\theta$. All the cases of HCMCs in this table verify the constraints of saturation (8) and effective use of window area (9). Table I contains also accepted and rejected cases with respect to the constraints of the minimum required $\mathrm{CM}$ inductance (10) and the stability of the converter (14). One can appreciate that most of the FEM simulation results conducted on each case present a good match with analytic results at $100 \mathrm{kHz}$. The first four cases 1,2,3,4 have all parameters fixed except ID. These cases are intended to study the effect of ID on $L_{C M}$ and $L_{\text {leak }}$. likewise, cases 4,5,6 and 7 are used to analyze OD effect on $L_{C M}$ and $L_{\text {leak }}$. The effect of $\mathrm{H}$ can be studied with the cases $7,8,9$, and 10 whereas cases 4 and 11 to 13 are used to study the effect of $\theta$. The range of cases where the number of turns $N=5$ was chosen among other ranges varying with $\mathrm{N}$ as it presents cases of which the input parameters (ID, OD, $\mathrm{H}$, and $\theta$ ) have very distant values which allow to clearly see their impact on $L_{C M}$ and $L_{\text {leak }}$. 
TABLE I: Samples of HCMCs estimated by the optimization algorithm

\begin{tabular}{|c|c|c|c|c|c|c|c|c|c|}
\hline \multirow{2}{*}{ Case } & \multirow{2}{*}{$\theta[\mathrm{deg}]$} & \multicolumn{3}{|c|}{ Core Dimension [mm] } & \multicolumn{2}{|c|}{ Calculated@100kHz } & \multicolumn{2}{|c|}{ Simulated@100kHz } & \multirow{2}{*}{ Status } \\
\hline & & $\mathrm{H}$ & OD & ID & $\mathrm{L}_{\mathrm{CM}}[\mu \mathrm{H}]$ & $\mathrm{L}_{\text {leak }}[\mu \mathrm{H}]$ & $\mathrm{L}_{\mathrm{CM}}[\mu \mathrm{H}]$ & $\mathrm{L}_{\text {leak }}[\mu \mathrm{H}]$ & \\
\hline 1 & $90^{\circ}$ & 5 & 25 & 22 & 29.144 & 1.66 & 29.17 & 1.642 & Refused $\left(L_{C M}<L_{C M_{-} r e q}\right)$ \\
\hline 2 & $90^{\circ}$ & 5 & 25 & 19 & 62.261 & 1.95 & 62.84 & 1.916 & Refused $\left(L_{C M}<L_{C M} M_{-} r e q\right)$ \\
\hline 3 & $90^{\circ}$ & 5 & 25 & 14 & 128.78 & 2.181 & 124.4 & 1.951 & Accepted \\
\hline 4 & $90^{\circ}$ & 5 & 25 & 7 & 256.8 & 2.300 & 292.5 & 2.297 & Accepted \\
\hline 5 & $90^{\circ}$ & 5 & 30 & 7 & 283.82 & 2.609 & 299.0 & 2.525 & Accepted \\
\hline 6 & $90^{\circ}$ & 5 & 18 & 7 & 200.86 & 1.786 & 200.9 & 1.756 & Accepted \\
\hline 7 & $90^{\circ}$ & 5 & 10 & 7 & 80.573 & 1.05 & 82.08 & 1.152 & Accepted \\
\hline 8 & $90^{\circ}$ & 10 & 25 & 7 & 513.655 & 2.765 & 573.1 & 2.600 & Refused $\left(L_{\text {leak }}>L_{D M_{-} \max } / 2\right)$ \\
\hline 9 & $90^{\circ}$ & 15 & 25 & 7 & 770.48 & 3.091 & 786.8 & 3.046 & Refused $\left(L_{\text {leak }}>L_{D M_{-} \max } / 2\right)$ \\
\hline 10 & $90^{\circ}$ & 20 & 25 & 7 & 1027 & 3.346 & 1153 & 3.342 & Refused $\left(L_{\text {leak }}>L_{D M \_\max } / 2\right)$ \\
\hline 11 & $110^{\circ}$ & 5 & 25 & 7 & 256.82 & 2.122 & 293.4 & 2.185 & Accepted \\
\hline 12 & $125^{\circ}$ & 5 & 25 & 7 & 256.82 & 2.038 & 292.2 & 2.009 & Accepted \\
\hline 13 & $135^{\circ}$ & 5 & 25 & 7 & 256.82 & 1.996 & 291.2 & 1.776 & Accepted \\
\hline
\end{tabular}

The simulation results for the variation of $L_{C M}$ and $L_{l e a k}$ with the core geometry and the angular coverage of its windings are illustrated in Fig. 6 and Fig. 7 respectively. The increase in ID introduces a decrease of $L_{C M}$ and $L_{\text {leak }}$ values whereas an increase in $\mathrm{OD}$ and $\mathrm{H}$ introduces their increase. The increase in $\theta$ does not affect $L_{C M}$ although smaller values of $\theta$ introduce a premature drop in $L_{C M}$ values at high frequencies which is the case when $\theta=90^{\circ}$. $L_{C M}$ value drops rapidly due to the small angular coverage of its core $\left(90^{\circ}\right)$ which force the winding of each phase to span a tight sector of the core reducing the interval between turns and increasing thereby the total turn-to-turn parasitic capacitances of the HCMC which leads to the saturation of its magnetic core. On the other hand, the increase of $\theta$ causes a decrease of the unwounded angle of the core and thus reduces $L_{\text {leak }}$ values.

The total number of accepted cases proposed by the optimization tool reached 782 cases after restricting the outputs values of the optimization algorithm namely $L_{\text {leak }}$ and V. These restrictions were set to reduce the number of generated cases and obtain the best possible results. The minimum value of $L_{l e a k}$ was set to $45.45 \%$ of $\mathrm{L}_{\mathrm{DM}} \_$max and $\mathrm{V}$ was set to a maximum value of $4 \mathrm{~cm}^{3}$. In the resulting intervals, $L_{\text {leak }}$ values cover a tight range $\left(2.273 \mu H<L_{l e a k}<2.499 \mu H\right)$, whereas the values of $L_{C M}$ and $\mathrm{V}$ span wide ranges respectively $\left(111.5 \mu H<L_{C M}<856.6 \mu H\right)$, $\left(0.6 \mathrm{~cm}^{3}<V<3.884 \mathrm{~cm}^{3}\right)$.

The resulting cases of HCMCs are sorted by the three parameters $L_{C M}, L_{\text {leak }}$, and $\mathrm{V}$ respectively. The cases with the highest $L_{C M}, L_{\text {leak }}$, and minimum $\mathrm{V}$ are favored. Table II present accepted cases in terms of $L_{C M}, L_{\text {leak }}$, and minimum volume. These prototypes are selected among the total accepted cases for implementation in the designed filter mainly because the values of their cores geometric parameters are very close to standard values of magnetic core models available in the market which ensure the possibility to validate the design procedure through future experimental tests. Through Table II, it can be noticed that the $\mathrm{CM}$ chokes corresponding to cases $\mathrm{A}$ and $\mathrm{C}$ owns respectively the largest sizes whereas cases $\mathrm{B}$ and $\mathrm{D}$ have almost similar sizes with the smallest size being attributed to case D. The simulation results of $L_{C M}$ and $L_{\text {leak }}$ for these cases shows a good agreement with analytical results at $100 \mathrm{kHz}$. These cases exhibit different values of $L_{C M}$ always above the required $\mathrm{CM}$ inductance as shown in Fig. 8a thus, improving the filter performances, while the leakage inductances $L_{\text {leak }}$ of these cases covers a small range with close values (Fig. 8b) despite the difference in their core's geometries and winding structures which confirm the strength of the proposed method to determine compact $\mathrm{CMCs}$ with effective $\mathrm{CM}$ inductances and adequate leakage inductances. 
TABLE II: The HCMCs proposed for implementation in the designed filter

\begin{tabular}{|c|c|c|c|c|c|c|c|c|c|c|}
\hline \multirow{2}{*}{ Case } & \multirow{2}{*}{$\theta$ [deg] } & \multirow{2}{*}{$\mathrm{N}$} & \multicolumn{3}{|c|}{ Core Dimension [mm] } & \multicolumn{2}{|c|}{ Calculated@100kHz } & \multicolumn{2}{|c|}{ Simulated@100 kHz } & \multirow{2}{*}{ Volume $\left[\mathrm{cm}^{3}\right]$} \\
\hline & & & $\mathrm{H}$ & OD & ID & $\mathrm{L}_{\mathrm{CM}}[\mu \mathrm{H}]$ & $\mathrm{L}_{\text {leak }}[\mu \mathrm{H}]$ & $\mathrm{L}_{\mathrm{CM}}[\mu \mathrm{H}]$ & $\mathrm{L}_{\text {leak }}[\mu \mathrm{H}]$ & \\
\hline A & $70^{\circ}$ & 5 & 7 & 20 & 10 & 213.535 & 2.299 & 224.3 & 2.294 & 3.151 \\
\hline $\mathrm{B}$ & $80^{\circ}$ & 6 & 4.7 & 15.8 & 8.9 & 173.023 & 2.319 & 178.1 & 2.323 & 1.498 \\
\hline $\mathrm{C}$ & $90^{\circ}$ & 6 & 6.3 & 16 & 9.6 & 207.556 & 2.385 & 212.6 & 2.384 & 1.922 \\
\hline $\mathrm{D}$ & $120^{\circ}$ & 7 & 5 & 14 & 9 & 194.967 & 2.449 & 198.0 & 2.483 & 1.261 \\
\hline
\end{tabular}

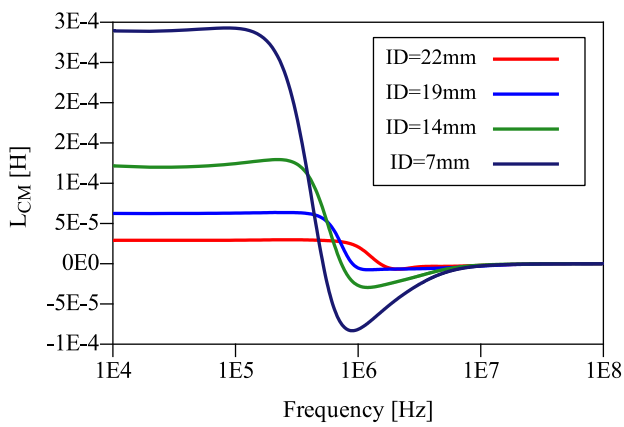

(a)
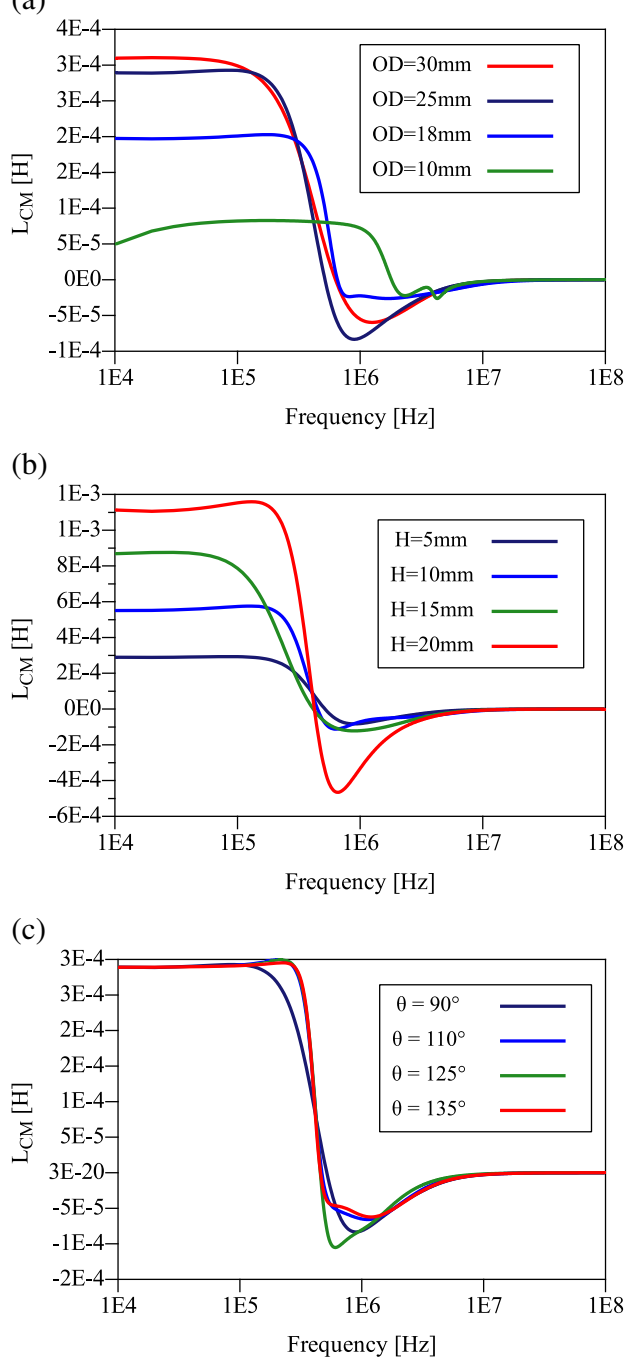

(d)

FIGURE 6: Variation of $L_{C M}$ with ID, OD, $\mathrm{H}$ and $\theta$ 63
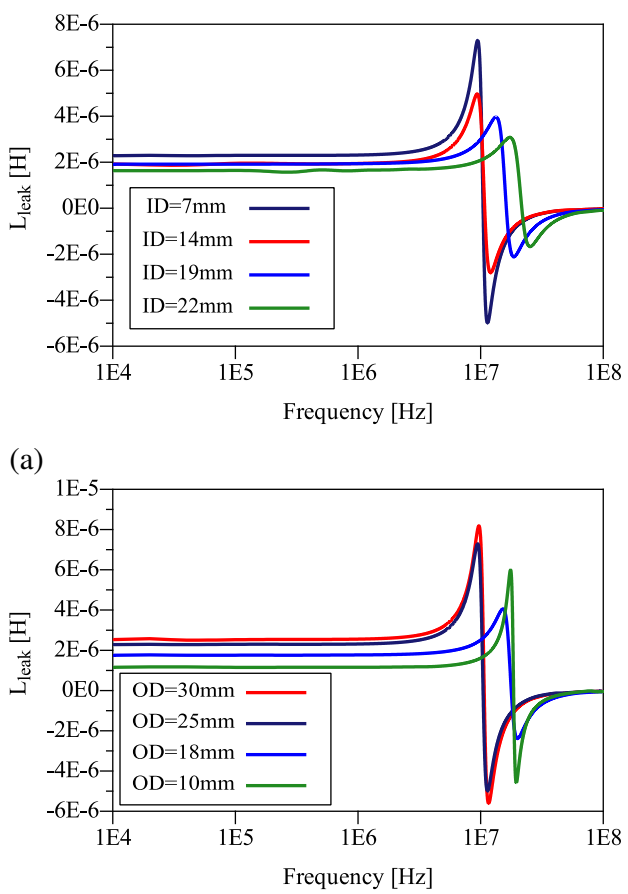

(b)

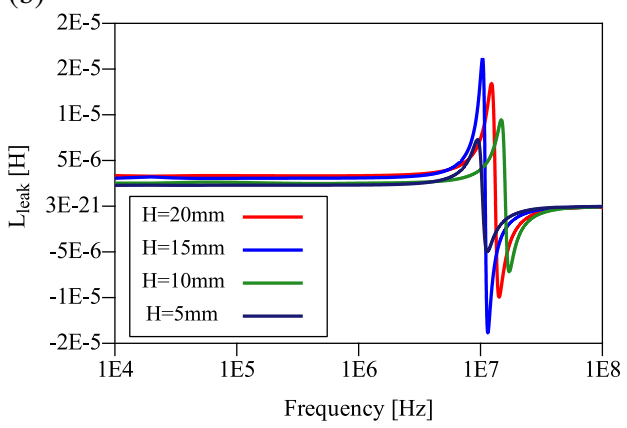

(c)

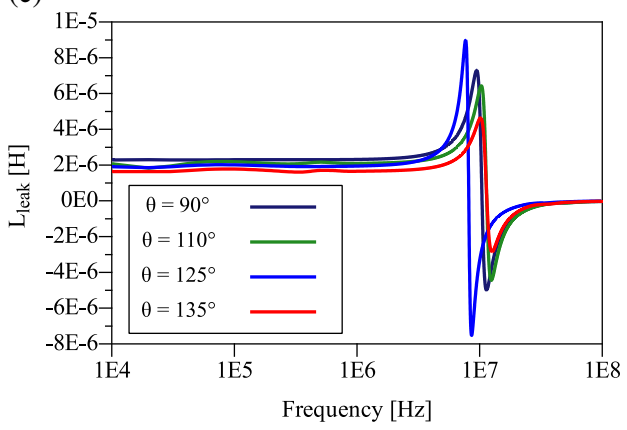

(d)

FIGURE 7: Variation of $L_{\text {leak }}$ with ID, OD, $\mathrm{H}$ and $\theta$ VOL. 10, NO. 2, JULY 2021 


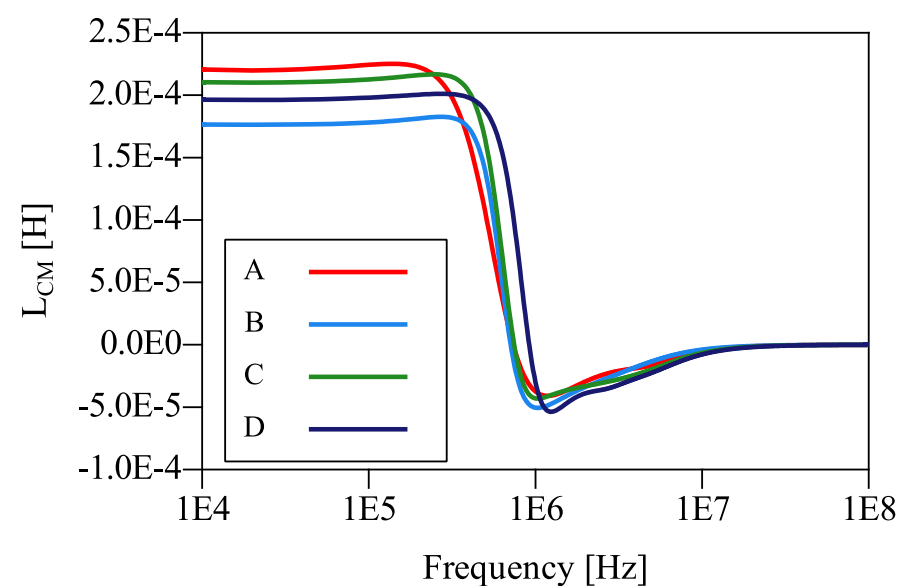

(a)

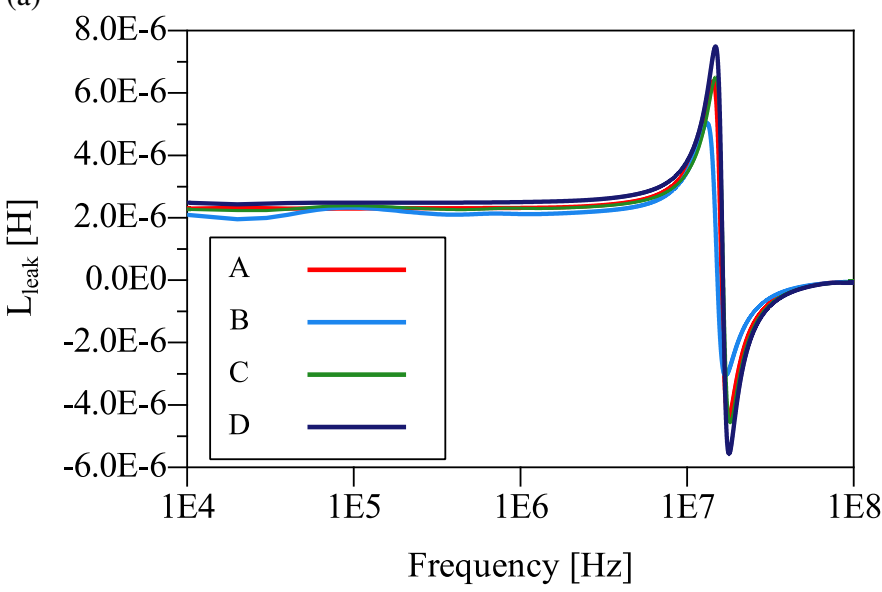

(b)

FIGURE 8: The CM inductances and Leakage inductances of the proposed cases A, B, C, D. (a) $L_{C M}$, (b) $L_{\text {leak }}$

\section{B. PERFORMANCE ANALYSIS OF THE PROPOSED HCMCS}

The filter CM and DM insertion loss (IL) profiles for each case of $\operatorname{HCMC}(\mathrm{A}, \mathrm{B}, \mathrm{C}, \mathrm{D})$ are illustrated in Fig. 9a and Fig. $9 \mathrm{~b}$ respectively. These profiles are affected by the behavior of these HCMCs respective $L_{C M}$ and $L_{\text {leak }}$. The CM IL profiles when using all four cases are tightly close at low frequencies then the disparity between them start slowly increasing from $600 \mathrm{kHz}$ till reaching its highest value of $33.7 \mathrm{~dB}$ at a frequency of $30.4 \mathrm{MHz}$ beyond the parasitic anti-resonance of the filter. On the other hand, the DM IL profiles keep almost the same allure till the parasitic anti-resonance at $22.22 \mathrm{MHz}$ where the difference beyond this point becomes higher $(8 \mathrm{~dB}$ at $50 \mathrm{MHz})$. In Fig. $8 \mathrm{a}$ we notice that $L_{C M}$ corresponding to case A which exhibits the highest value at low frequency (till $250 \mathrm{kHz}$ ) owing to its core superior size, starts dropping rapidly due to the small angular coverage of its core $\left(70^{\circ}\right)$. This decrease causes the degradation of the filter CM IL beyond $600 \mathrm{kHz}$ as shown in Fig. 9a. The CM IL profiles of the filter when using the HCMC of cases B and C are very close however the CM IL profile of case B is slightly degraded compared to that of case $\mathrm{C}$ given the small size and inferior angular coverage of its magnetic core. Nonetheless, the CM IL profiles of the filter when using case $\mathrm{B}$ and $\mathrm{C}$ shows on their part a better response than that of case $\mathrm{A}$ beyond $2 \mathrm{MHz}$. This is due to their cores wider angular coverages which diminish the excitation of their turn-to-turn parasitic capacitances at high frequencies. For its part, the filter using the HCMC of case $\mathrm{D}$ presents the best CM IL in practically the entire range of $(700 \mathrm{KHz}-70 \mathrm{MHz})$. This is owing to the constant value of its CM inductance sustained up to around $450 \mathrm{kHz}$. These performances are reached by compensating its core small size by a combination of an increased number of turns and superior angular coverage. Fig. 10 reflects the impact of the proposed HCMCs on the filter performances against the conducted EMI induced by the considered power converter. All the cases of optimized HCMCs including the smallest one corresponding to case D allow the EMI filter to easily qualify the power converter to EMC requirements since its both CM and DM conducted EMI meets the limit of the standard over the full range $(150 \mathrm{KHz}-30 \mathrm{MHz})$.

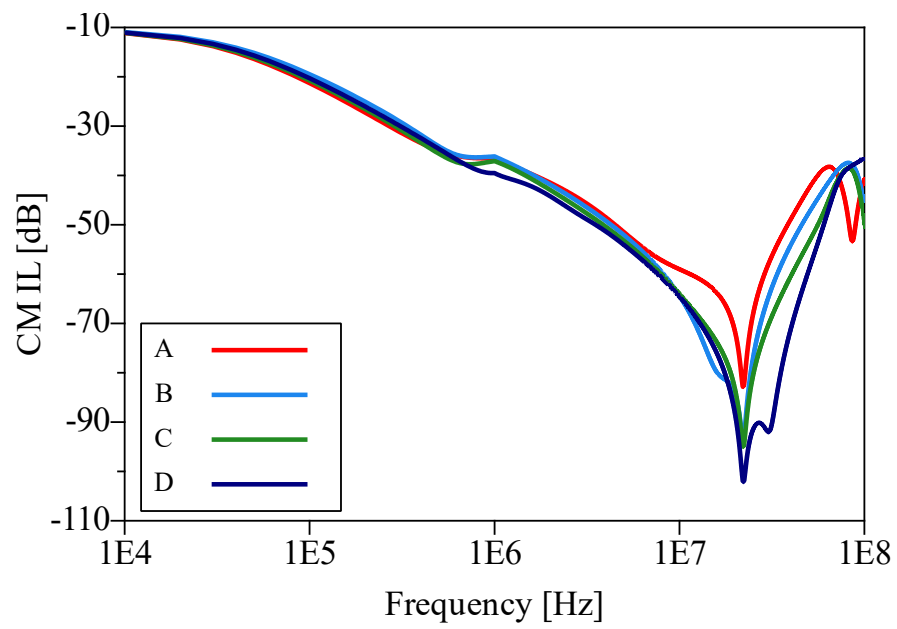

(a)

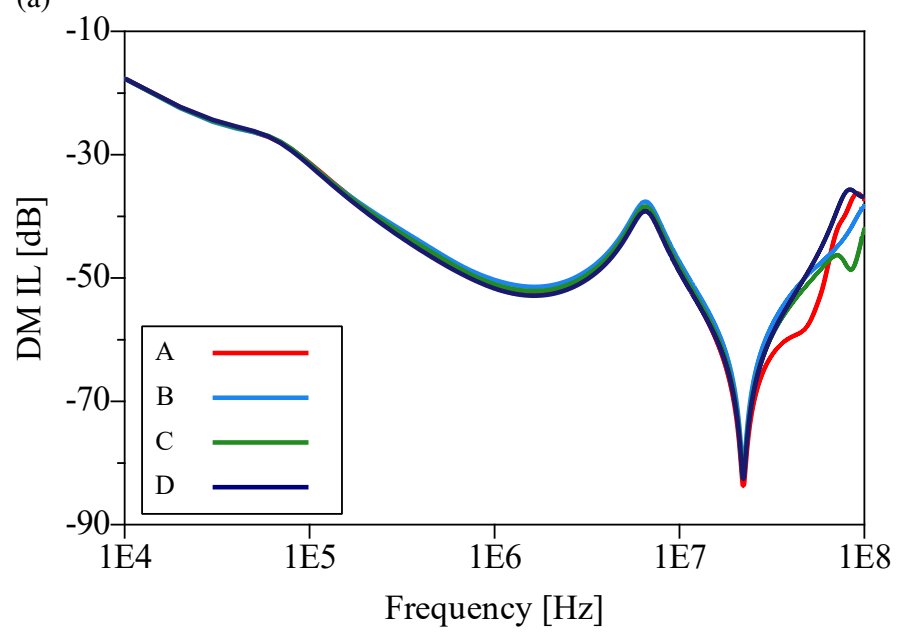

(b)

FIGURE 9: The filter insertion loss using the proposed cases of HCMCs. (a) CM insertion loss, (b) DM insertion loss 


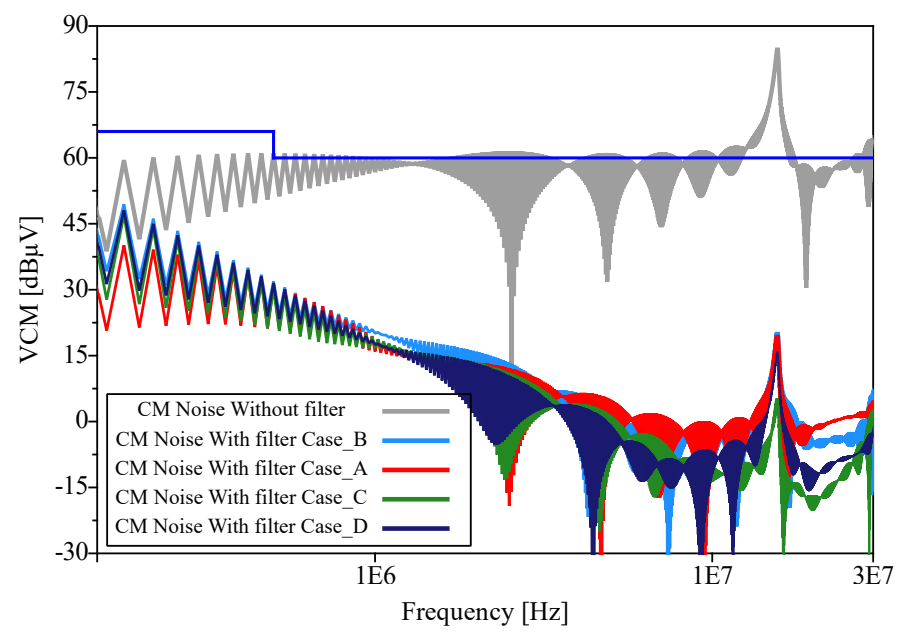

(a)

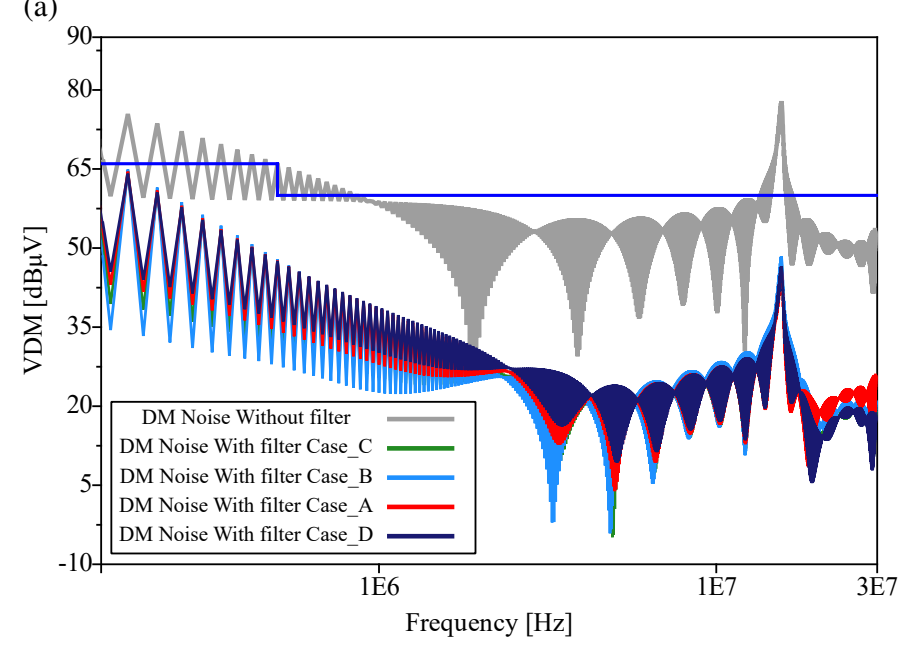

(b)

FIGURE 10: The mitigation performances of the filter against the conducted CM and DM EMI using the proposed cases of HCMCs. (a) CM EMI, (b) DM EMI

\section{CONCLUSION}

In this paper, an effective optimization method of toroidal hybrid CM chokes for EMI filter design is presented. The optimization method is based on rule-based variations of these chokes' magnetic cores dimensions and winding structures taking into account several constraints related to their physical and geometrical limits as well as the constraints related to the EMI filter design. The strength of the optimization method has been verified with respect to these predefined constraints. 3D FEM simulations of proposed cases were performed and compared with analytic calculations. The proposed optimization method allows through a small computational task to propose a variety of configurations for compact HCMCs that delivers effective CMCs with optimized leakage inductances improving by this both the power density and the filtering performances of the designed EMI filter. Future works objectives will focus on the enhancement of the proposed method by including constraints on additional parameters of the CM choke such as the parasitic capacitance and thermal issues related to the losses in the windings.

\section{REFERENCES}

[1] B. Narayanasamy, H. Peng, Z. Yuan, A. I. Emon, and F. Luo, "Modeling and Analysis of a Differential Mode Active EMI Filter with an Analog Twin Circuit," IEEE Trans. Electromagn. Compat., vol. 62, no. 4, pp. 1591-1600, 2020, doi: 10.1109/TEMC.2020.3006427.

[2] R. Lai, Y. Maillet, F. Wang, S. Wang, R. Burgos, and D. Boroyevich, "An integrated EMI choke for differential-mode and common-mode noise suppression," IEEE Trans. Power Electron., vol. 25, no. 3, pp. 539-544, 2010, doi: 10.1109/TPEL.2009.2030803.

[3] W. Tan, C. Cuellar, X. Margueron, and N. Idir, "A common-mode choke using toroid-EQ mixed structure,” IEEE Trans. Power Electron., vol. 28, no. 1, pp. 31-35, 2013, doi: 10.1109/TPEL.2012.2205708.

[4] F. Luo, D. Boroyevich, P. Mattevelli, K. Ngo, D. Gilham, and N Gazel, "An integrated common mode and differential mode choke for EMI suppression using magnetic epoxy mixture," Conf. Proc. - IEEE Appl. Power Electron. Conf. Expo. - APEC, pp. 1715-1720, 2011, doi: 10.1109/APEC.2011.5744827.

[5] Y. Liu, S. Jiang, W. Liang, H. Wang, and J. Peng, "Modeling and Design of the Magnetic Integration of Single-and Multi-Stage EMI Filters," IEEE Trans. Power Electron., vol. 35, no. 1, pp. 276-288, 2020, doi: 10.1109/TPEL.2019.2915804.

[6] B. Zaidi, A. Videt, and N. Idir, "Optimization Method of CM Inductor Volume Taking into Account the Magnetic Core Saturation Issues," IEEE Trans. Power Electron., vol. 34, no. 5, pp. 4279-4291, 2019, doi: 10.1109/TPEL.2018.2861620.

[7] J. L. Kotny, X. Margueron, and N. Idir, "High-frequency model of the coupled inductors used in EMI filters," IEEE Trans. Power Electron., vol. 27, no. 6, pp. 2805-2812, 2012, doi: 10.1109/TPEL.2011.2175452.

[8] L. Fang, D. Boroyevich, P. Mattevelli, and N. Gazel, "A comprehensive design for high power density common mode EMI inductor," IEEE Energy Convers. Congr. Expo. Energy Convers. Innov. a Clean Energy Futur. ECCE 2011, Proc., pp. 1861-1867, 2011, doi: 10.1109/ECCE.2011.6064012.

[9] M. L. Heldwein, L. Dalessandro, and J. W. Kolar, "The threephase common-mode inductor: Modeling and design issues," IEEE Trans. Ind. Electron., vol. 58, no. 8, pp. 3264-3274, 2011, doi: 10.1109/TIE.2010.2089949.

[10] D. Han, C. T. Morris, W. Lee, and B. Sarlioglu, "Three-phase common mode inductor design and size minimization," 2016 IEEE Transp. Electrif. Conf. Expo, ITEC 2016, no. January 2018, 2016, doi: 10.1109/ITEC.2016.7520267.

[11] G. Ala, G. C. Giaconia, G. Giglia, M. C. Di Piazza, and G. Vitale, "Design and performance evaluation of a high power density EMI filter for PWM inverter-fed induction motor drives," 2015 IEEE 15th Int. Conf. Environ. Electr. Eng. EEEIC 2015 - Conf. Proc., pp. 1573-1579, 2015, doi: 10.1109/EEEIC.2015.7165406.

[12] J. L. Kotny, T. Duquesne, and N. Idir, "Influence of the common mode impedance paths on the design of the EMI filters used with SiCbuck converter," Adv. Electromagn., vol. 4, no. 2, pp. 44-53, 2015, doi: 10.7716/aem.v4i2.281

[13] G. Ala et al., "Computer aided optimal design of high power density EMI filters,” EEEIC 2016 - Int. Conf. Environ. Electr. Eng., 2016, doi 10.1109/EEEIC.2016.7555572.

[14] G. Ala et al., "Optimized design of high power density EMI filters for power electronic converters," AEIT 2016 - Int. Annu. Conf. Sustain. Dev. Mediterr. Area, Energy ICT Networks Futur., 2016, doi 10.23919/AEIT.2016.7892794.

[15] G. Giglia et al., "Automatic EMI filter design for power electronic converters oriented to high power density," Electron., vol. 7, no. 1, 2018, doi 10.3390/electronics7010009.

[16] G. Giglia, G. Ala, L. Mistretta, G. C. Giaconia, S. Quondam Antonio, and H. P. Rimal, "Design and Assessment of Optimized EMI Filters for Avionic Applications," IEEE 4th Int. Forum Res. Technol. Soc. Ind. RTSI 2018 - Proc., pp. 1-6, 2018, doi: 10.1109/RTSI.2018.8548440.

[17] C. Jettanasen and A. Ngaopitakkul, "The conducted emission attenuation of micro-inverters for nanogrid systems," Sustain., vol. 12, no. 1, pp. 1-32, 2020, doi: 10.3390/SU12010151.

[18] M. Luna, G. La Tona, A. Accetta, M. Pucci, and M. C. Di Piazza, "An evolutionary EMI filter design approach based on in-circuit insertion loss 
and optimization of power density," Energies, vol. 13, no. 8, 2020, doi: 10.3390/en13081957.

[19] A. Roc'h and F. Leferink, "Towards a first time right design of the common mode choke," 2011 30th URSI Gen. Assem. Sci. Symp. URSIGASS 2011, pp. 8-11, 2011, doi: 10.1109/URSIGASS.2011.6050765.

[20] A. Roc'h and F. Leferink, "Optimization of the in situ Performance of Common Mode Chokes for Power Drive Systems Using Designable Parameters," IEEE Trans. Electromagn. Compat., vol. 60, no. 2, pp. 1-7, 2018, doi: 10.1109/TEMC.2017.2716384.

[21] A. Roc'H and F. Leferink, "Contributing factors in the final performance of a common mode choke," IEEE Int. Symp. Electromagn. Compat., vol. 2015-Septm, pp. 120-123, 2015, doi: 10.1109/ISEMC.2015.7256143.

[22] D. Varajão, R. E. Araújo, L. M. Miranda, and J. A. P. Lopes, "EMI filter design for a single-stage bidirectional and isolated ac-dc matrix converter," Electron., vol. 7, no. 11, pp. 1-21, 2018, doi: 10.3390/electronics7110318.

[23] K. Nomura, T. Kojima, A. Takahashi, Y. Hattori, and K. Torii, "Conducted common mode noise reduction for boost converters using leakage inductance of coupled inductor," Conf. Proc. - IEEE Appl. Power Electron. Conf. Expo. - APEC, no. Dm, pp. 294-299, 2017, doi: 10.1109/APEC.2017.7930708.

[24] Y. Liu et al., "FEM modelling of three-phase common mode choke for performance evaluation," 2016 Asia-Pacific Int. Symp. Electromagn. Compat. APEMC 2016, pp. 96-99, 2016, doi: 10.1109/APEMC.2016.7522921.

[25] F. De León, S. Purushothaman, and L. Qaseer, "Leakage inductance design of toroidal transformers by sector winding," IEEE Trans. Power Electron., vol. 29, no. 1, pp. 473-480, 2014, doi: 10.1109/TPEL.2013.2251429.

[26] M. J. Nave, "On modeling the common mode inductor," IEEE Int. Symp. Electromagn. Compat., pp. 452-457, 1991, doi: 10.1109/isemc.1991.148275.

[27] N. Benhadda, A. Bendaoud, and N. Chikhi, "A conducted EMI noise prediction in DC/DC converter using a frequency-domain approach," Elektroteh. Vestnik/Electrotechnical Rev., vol. 85, no. 3, pp. 103-108, 2018. 